\title{
Facet-embedded thin-film III-V edge-emitting lasers integrated with SU-8 waveguides on silicon
}

\author{
Sabarni Palit, ${ }^{1, *}$ Jeremy Kirch, ${ }^{2}$ Mengyuan Huang, ${ }^{1}$ Luke Mawst, ${ }^{2}$ and Nan Marie Jokerst ${ }^{1}$ \\ ${ }^{1}$ Department of Electrical and Computer Engineering, Duke University, Box 90291, Durham, North Carolina 27708, USA \\ ${ }^{2}$ Department of Electrical and Computer Engineering, University of Wisconsin-Madison, \\ 1415 Engineering Drive, Madison, Wisconsin 53706-1691, USA \\ *Corresponding author: sabarni.palit@duke.edu
}

Received August 31, 2010; accepted September 2, 2010;

posted September 27, 2010 (Doc. ID 134169); published October 13, 2010

\begin{abstract}
A thin-film InGaAs/GaAs edge-emitting single-quantum-well laser has been integrated with a tapered multimode SU-8 waveguide onto an Si substrate. The SU-8 waveguide is passively aligned to the laser using mask-based photolithography, mimicking electrical interconnection in Si complementary metal-oxide semiconductor, and overlaps one facet of the thin-film laser for coupling power from the laser to the waveguide. Injected threshold current densities of $260 \mathrm{~A} / \mathrm{cm}^{2}$ are measured with the reduced reflectivity of the embedded laser facet while improving single mode coupling efficiency, which is theoretically simulated to be $77 \%$. (C) 2010 Optical Society of America

OCIS codes: $130.3120,130.5460,130.6622,230.3120,250.3140,250.5300$.
\end{abstract}

The planar integration of photonic components onto postprocessed Si or Si complementary metal-oxide semiconductor platforms have applications ranging from optical interconnects to environmental/biological/chemical sensing [1-6]. A basic building block of these planar photonic chip-scale systems is a thin-film III-V edgeemitting laser (TF-EEL) integrated with a waveguide on the same substrate. To realize low-power, portable, cost-effective chip-scale integrated systems, the laser power consumption must be minimized, the laser to waveguide coupling maximized, and the process technology simplified. Thus, the laser threshold current density, $J_{\text {th }}$, should be low, the waveguide should be optimally coupled with the laser with minimal alignment requirements, and efficient heat sinking strategies should be implemented.

Integration of TF-EELs with waveguides has been demonstrated on $\mathrm{SiO}_{2} / \mathrm{Si}$ [7], silicon-on-insulator [8,9], and with polymers [10-12], in addition to the characterization of photodetectors with polymer waveguides, for both $\mathrm{Si}$ photodiodes [13] and thin-film III-V metal-semiconductor metal photodetectors $[11,14]$. In [8-11], both $p$ and $n$ contacts are on the top of the laser, compromising current flow, and the interface between the laser and the substrate is thermally insulating, compromising thermal management. In [7], the TF-EELs are placed in a trench in a slab waveguide, requiring precise control over etch depth and vertical alignment. Likewise, in [8], the simple cleaved hybrid laser has critical alignment requirements with other optical components. The TF-EELs end-fire coupled to waveguides [7,9-12] all have an air gap between the laser and the waveguide, except for [9] , in which the etched, not cleaved, EEL facet is embedded in the waveguide.

In this Letter, we present the planar integration of a $980 \mathrm{~nm}$ TF-EEL with one cleaved facet embedded in a multimode $\mathrm{SU}-8$ waveguide on $\mathrm{a} \mathrm{SiO}_{2} / \mathrm{Si}$ substrate. This TF-EEL has top and bottom metal contacts, a $p$-side ridge, an $n$ metal stripe for low $J_{\text {th }}$ operation, and a thermally conducting broad area metal contact bond to the
$\mathrm{SiO}_{2} / \mathrm{Si}$ integration substrate. There is no gap between the cleaved TF-EEL and the SU-8 waveguide, increasing the coupling efficiency in comparison to systems with a gap. In contrast to previously reported work $[\underline{15}, 16]$, in a new sample reported herein, the laser facet was embedded directly in the waveguide after bonding to the $\mathrm{Si}$. In addition, a new detailed analysis and simulation of this and other samples reported in [16] is presented for the first time herein. Passive alignment between the laser and waveguide is realized through mask-based photolithography, thus enabling the definition of wafer scale passively aligned waveguide optical interconnections. A metal pad for the laser is deposited onto the $\mathrm{Si}$ substrate. The laser is aligned and bonded to the metal pad, and the $n$ stripe and fiducial marks are patterned. The waveguides are aligned passively to the laser by aligning the waveguides to the fiducial marks, which can be expanded to all integrated optical components, to the wafer scale. However, the embedding of the laser facet also reduces its Fresnel reflectivity, increasing the mirror loss. In this Letter, lasing has been demonstrated with this lower facet reflectivity, resulting in the lowest $J_{\text {th }}\left(260 \mathrm{~A} / \mathrm{cm}^{2}\right)$ reported to date for a facet-embedded TF-EEL.

The embedded TF-EELs are composed of an $\mathrm{In}_{0.2} \mathrm{Ga}_{0.8} \mathrm{As} / \mathrm{GaAs}$ single-quantum-well separate confinement heterostructure, strain compensated with tensile $\mathrm{GaAs}_{0.7} \mathrm{P}_{0.3}$ barrier layers, and they are index-guided EELs with cleaved facets. The material growth, fabrication, and integration are detailed in [17]. Lasers of cavity lengths $800 \mu \mathrm{m}$ and $1000 \mu \mathrm{m}$ were cleaved and integrated onto a $4 \mathrm{~mm} \times 4 \mathrm{~mm} \quad \mathrm{Ti}(30 \mathrm{~nm}) / \mathrm{Pt}(40 \mathrm{~nm}) /$ $\mathrm{Au}(200 \mathrm{~nm})$ metal pad on $\mathrm{SiO}_{2} / \mathrm{Si}$. The lasers have $p$ ridges that are $50 \mu \mathrm{m}$ wide and $1.01 \mu \mathrm{m}$ deep with $40 \mu \mathrm{m} n$ stripes. The front facet of the laser is integrated close to the edge of the metal bonding pad to prevent reflections from the pad, as shown in Fig. 1. After the SU-8-2002 was spun onto the integrated laser/Si platform at a thickness of $2.6 \mu \mathrm{m}$, it was patterned with standard photolithography to form a tapered waveguide that 


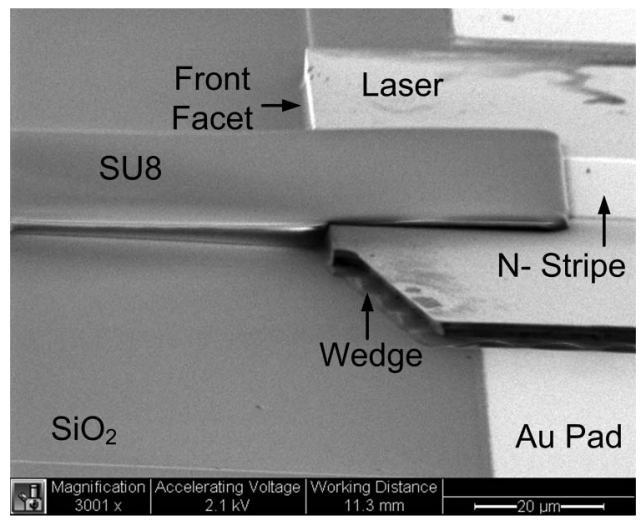

Fig. 1. Scanning electron micrograph (SEM) image of embedded TF-EEL facet and SU-8 waveguide. The TF-EEL bar is preferentially cleaved along the wedge shown in the SEM image.

overlapped one cleaved facet of the TF-EEL. This linear taper narrows from 86 to $8 \mu \mathrm{m}$ over a $1.6 \mathrm{~mm}$ length.

The integrated EEL/waveguide system was tested using a $1 \mathrm{kHz}, 0.01 \%$ duty cycle pulsed drive current input to the EEL, and the output light was collected from the end of the waveguide with a multimode fiber (positioned to maximize collection) connected to an HP 8163A power meter with an HP 81521B optical head. Figure 2(a) shows the normalized waveguide power versus $\overline{\mathrm{EEL}}$ drive current (L-I) characteristics of an 800- $\mu$ m-long laser inte-
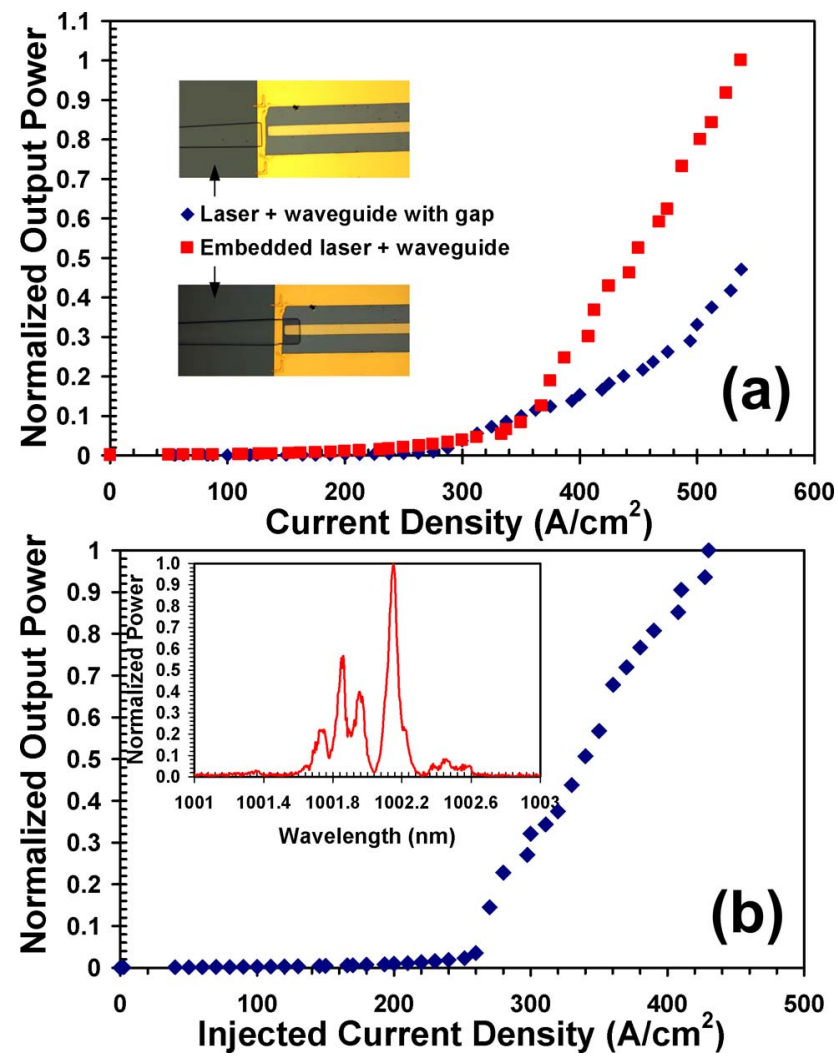

Fig. 2. (Color online) (a) Waveguide output power versus current (L-I) from 800- $\mu$ m-long laser integrated with an SU-8 waveguide. The original integration (blue) with gap has lower $J_{\text {th }}$ and lower power. After embedding (red), the L-I has a $27.2 \%$ higher $J_{\text {th }}$ and higher coupled power. (b) L-I for an embedded facet $1000 \mu \mathrm{m}$ laser, showing $J_{\text {th }}=260 \mathrm{~A} / \mathrm{cm}^{2}$. Inset, spectrum at $J=420 \mathrm{~A} / \mathrm{cm}^{2}$. grated with a tapered waveguide with both separated and facet-embedded configurations. In this sample, the laser extends approximately $24 \mu \mathrm{m}$ from the pad edge. The EEL was first tested with a waveguide that did not embed the EEL facet. For this case, the L-I characteristic indicates a threshold current of $110 \mathrm{~mA} \quad\left(J_{\mathrm{th}}=\right.$ $275 \mathrm{~A} / \mathrm{cm}^{2}$ ). An overlapping waveguide was then patterned onto the original waveguide, leading to a threshold current of $140 \mathrm{~mA}\left(J_{\mathrm{th}}=350 \mathrm{~A} / \mathrm{cm}^{2}\right)$. This $27.2 \%$ change in threshold current is a result of the reduced front facet reflectivity due to the addition of the polymer to the EEL facet. The slope efficiencies are not necessarily comparable, as the shape of the overlapping waveguide is modified by patterning on top of the existing waveguide. A clearer comparison is obtained from simulations. Figure 2(b) shows a typical L-I and spectral characteristic for these embedded facet lasers, using a $1000-\mu \mathrm{m}$-long laser with $J_{\text {th }}=260 \mathrm{~A} / \mathrm{cm}^{2}$ [16]. Heating of the contacts prevented $\mathrm{cw}$ measurements, which can be overcome by packaging the laser and optimized processing of the metal contacts. The lasing operation was also confirmed by measurements up to $60^{\circ} \mathrm{C}$, and finite-element-method (FEM) simulations indicated a $47^{\circ} \mathrm{C}$ rise in junction temperature for $\mathrm{cw}$ operation at $300 \mathrm{~K}$ on an Si substrate [18].
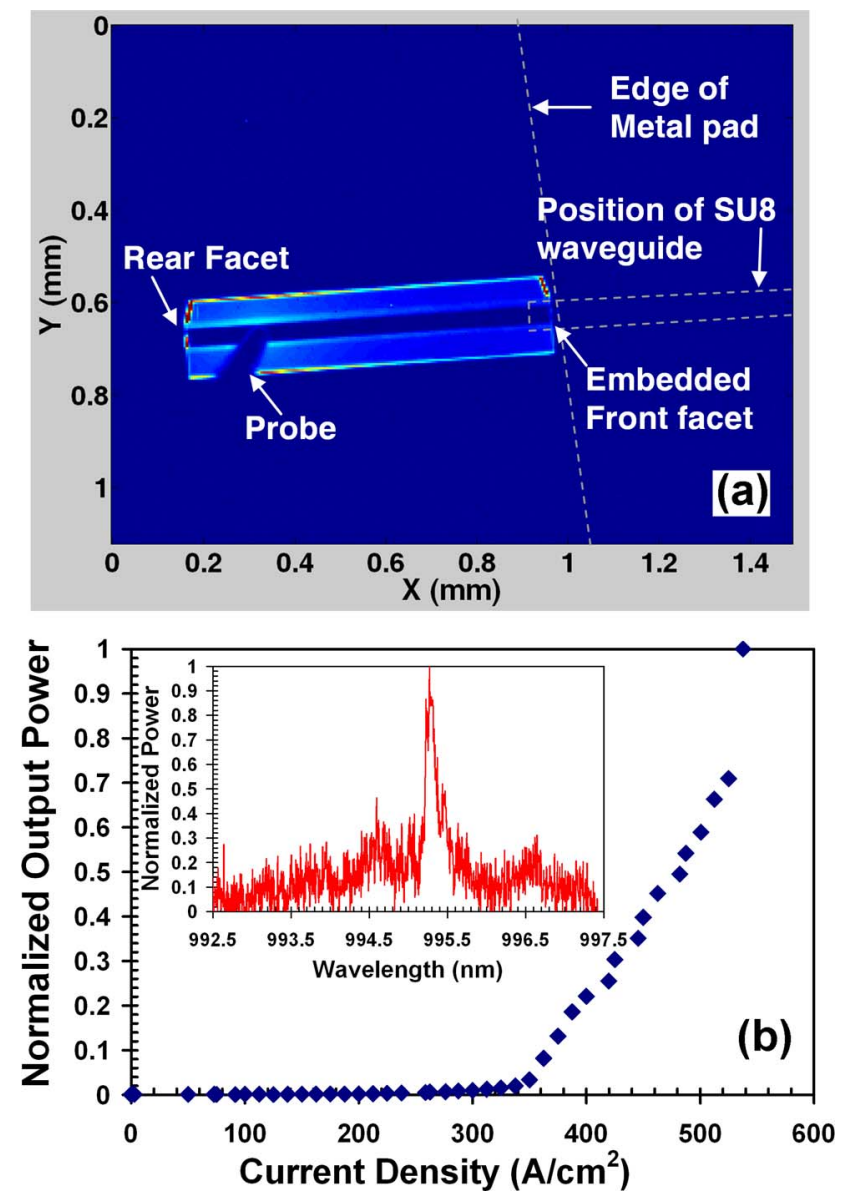

Fig. 3. (Color online) (a) IR image of an $800 \mu \mathrm{m}(40 \mu \mathrm{m} p$ metal stripe, $p$ ridge width of $50 \mu \mathrm{m}$ and depth of $0.61 \mu \mathrm{m}$, and $n$ metal stripe of $40 \mu \mathrm{m}$ ) laser with front facet embedded in SU-8 tapered $\mathrm{S}$ bend waveguide of $4 \mu \mathrm{m}$ width. (b) L-I characteristic and spectrum for the system. 


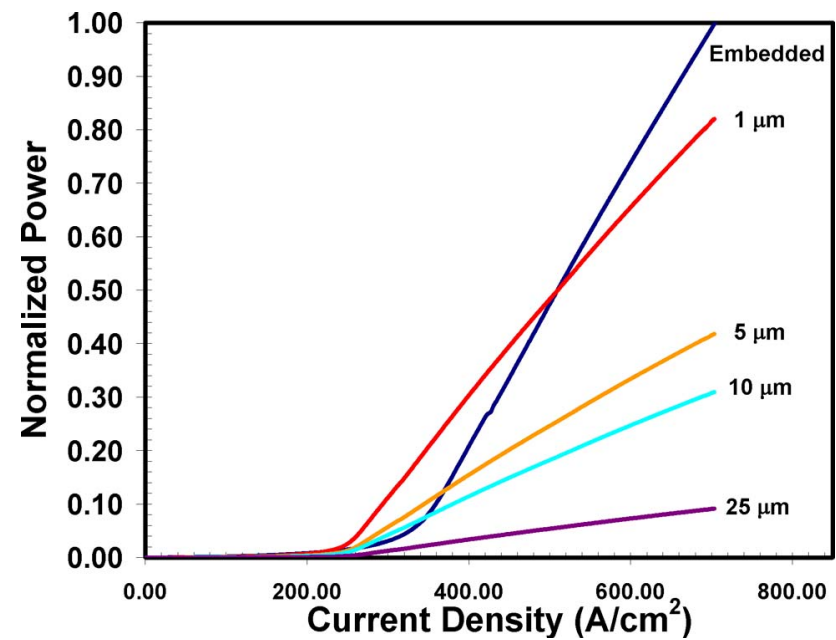

Fig. 4. (Color online) Simulated waveguide power as a function of injected current density for the embedded and separated cases. $J_{\text {th }}$ increases by $33 \%$, but the slope efficiency is higher.

A system in which the waveguide initially embedded the laser facet was measured for an $800 \mu \mathrm{m}$ laser with a ridge depth of $0.61 \mu \mathrm{m}$. A tapered $\mathrm{S}$ bend multimode waveguide was used to reduce the possibility of detecting a substrate mode, which consisted of two $90^{\circ}$ bends of $4 \mathrm{~mm}$ radii. Figure 3(a) shows an IR image of the top view of this integrated laser and waveguide. Dashed gray lines indicate the positions of the various components. $J_{\text {th }}$ is $357 \mathrm{~A} / \mathrm{cm}^{2}$, and $\lambda=995.27 \mathrm{~nm}$, as shown in Fig. 3(b).

The integrated EEL was simulated using Silvaco ATLAS. The front facet Fresnel calculated reflectivity changed from $31.3 \%$ to $14.7 \%$ due to the SU-8 waveguide embedding the facet. Simulations indicate that this resulted in a $33 \%$ increase in $J_{\text {th }}$, which compares well with the $27.2 \%$ increase observed experimentally. The coupling of the EEL to the waveguide as a function of separation was simulated by 2D FEM using COMSOL [15]; however, this simulation was limited to a single transverse mode. Figure 4 shows the simulated waveguide power versus EEL injected current density for 1, 5, 10, and $25 \mu \mathrm{m}$ separations. The light incident on the waveguide facet (values in [15]) has a fraction lost to the air and $\mathrm{SiO}_{2}$ claddings due to the high divergence. The calculated facet-embedded coupling efficiency from the laser to the waveguide for a single transverse mode of the laser, and for a waveguide mode power at distance of $140 \mu \mathrm{m}$ from the laser facet, is $77 \%$. These simulations also indicate higher slope efficiency from the embedded facet EEL, again due to reduced facet reflectivity.

In conclusion, facet-embedded TF-EELs integrated with $\mathrm{SU}-8$ polymer waveguides on $\mathrm{SiO}_{2} / \mathrm{Si}$ have been ex- perimentally characterized and theoretically analyzed with good agreement.

The authors would like to thank Sulochana Dhar for assistance with simulations and the United States Army Research Office (USARO) for supporting this work through ARO MURI W911NF-05-1-0262.

\section{References}

1. D. A. B. Miller, Proc. IEEE 88, 728 (2000).

2. Y. J. Joo, S. Fike, K. S. Chung, M. Brooke, N. M. Jokerst, and D. S. Wills, in Proceedings of the Fourth International Conference on Massively Parallel Processing Using Optical Interconnections (IEEE, 1997), pp. 96-100.

3. R. E. Kunz, Sens. Act. B 38, 13 (1997).

4. J. A. Chediak, Z. Luo, J. Seo, N. Cheung, L. P. Lee, and T. M. Sands, Sens. Act. A 111, 1 (2004).

5. J. Hubner, T. Anhøj, S. Pedersen, D. A. Zauner, A. M. Jorgensen, G. Blagoi, I. Talian, and O. Hansen, Proc. SPIE 6896, 689614 (2008).

6. N. Jokerst, M. Royal, S. Palit, L. Luan, S. Dhar, and T. Tyler, J. Biophoton. 2, 212 (2009).

7. M. Yanagisawa, H. Terui, K. Shuto, T. Miya, and M. Kobayashi, IEEE Photon. Technol. Lett. 4, 21 (1992).

8. R. Jones, H. D. Park, A. W. Fang, J. E. Bowers, O. Cohen, O. Raday, and M. J. Paniccia, J. Mat. Sci. Mat. Electron. 20, 3 (2007).

9. G. Roelkens, J. Brouckaert, S. Verstuyft, J. Schrauwen, D. Van Thourhout, and R. Baets, in Proceedings of the Third IEEE International Conference on Group IV Photonics (IEEE, 2006), pp. 188-190.

10. P. M. DeDobbelaere, F. Vermaerke, G. Vermeire, P. Demeester, P. VanDaele, G. R. Moehlmann, J.-L. P. Heideman, and W. H. G. Horsthuis, Proc. SPIE 2285, 352 (1994).

11. S.-W. Seo, S.-Y. Cho, and N. M. Jokerst, IEEE Photon. Technol. Lett. 17, 2197 (2005).

12. H.-F. Kuo, S.-Y. Cho, J. Hall, and N. M. Jokerst, in Proceedings of the 16th Annual Meeting of the IEEE Lasers and Electro-Optics Society (IEEE, 2003), pp. 63-64.

13. P. de la Fuente, J. A. Etxeberria, J. Berganzo, J. M. Ruano-López, M. T. Arroyo, E. Castaño, and F. J. Gracia, Sens. Act. A 123-124, 313 (2005).

14. S.-Y. Cho, S.-W. Seo, N. M. Jokerst, and M. Brooke, in Proceedings of the 55th Electronic Components Technology Conference (2005), pp. 1611-1616.

15. S. Palit, J. Kirch, L. Mawst, T. Kuech, and N. M. Jokerst, Proc. SPIE 7607, 76070I (2010).

16. S. Palit, J. Kirch, L. Mawst, and N. M. Jokerst, in Proceedings of the Conference on Lasers and Electro-Optics (Optical Society of America, 2010), paper CWP5.

17. S. Palit, J. Kirch, G. Tsvid, L. Mawst, T. Kuech, and N. M. Jokerst, Opt. Lett. 34, 2802 (2009).

18. S. Palit, J. Kirch, L. Mawst, T. Kuech, and N. M. Jokerst, "Thermal characteristics of III/V thin film edge emitting lasers on silicon," submitted to Int. J. Microw. Opt. Tech. 